\title{
Single-Isocenter Frameless Volumetric Modulated Arc Radiosurgery For Multiple Intracranial Metastases
}

\author{
Steven K. M. Lau, M.D., Ph.D. ${ }^{1}$, Kaveh Zakeri, M.D. ${ }^{1}$, Xiao Zhao, M.D. ${ }^{1}$, Ruben Carmona, \\ M.D. ${ }^{1}$, Erik Knipprath, B.A. ${ }^{2}$, Daniel R. Simpson, M.D. ${ }^{1}$, Sameer K. Nath, M.D. ${ }^{3}$, Gwe-Ya Kim, \\ Ph.D. ${ }^{1}$, Parag Sanghvi, M.D. ${ }^{1}$, Jona A. Hattangadi-Gluth, M.D. ${ }^{1}$, Clark C. Chen, M.D., Ph.D. \\ 2, ${ }^{*}$, and Kevin T. Murphy, M.D. ${ }^{1, *}$ \\ ${ }^{1}$ Department of Radiation Medicine and Applied Sciences, Moores University of California San \\ Diego Cancer Center, La Jolla, California, USA \\ ${ }^{2}$ Department of Surgery, Division of Neurosurgery, Moores University of California San Diego \\ Cancer Center, La Jolla, California, USA \\ ${ }^{3}$ Department of Therapeutic Radiology, Yale University School of Medicine, New Haven, \\ Connecticut, USA
}

\section{Abstract}

Background-Stereotactic radiosurgery is a well-accepted treatment for patients with intracranial metastases, but outcomes with volumetric modulated arc radiosurgery (VMAR) are poorly described.

Objective-To report our initial clinical experience applying a novel single-isocenter technique to frameless VMAR for simultaneous treatment of multiple intracranial metastases.

Methods-We performed a retrospective analysis of 15 patients undergoing frameless VMAR for multiple intracranial metastases using a single, centrally-located isocenter between 2009 and 2011. Among these, 3 patients were treated for progressive or recurrent intracranial disease. A total of 62 metastases (median 3 per patient, range 2-13) were treated to a median dose of $20 \mathrm{~Gy}$ (range, 15-30 Gy). 3 patients were treated with fractionated SRS. Follow-up including clinical examination and magnetic resonance imaging (MRI) occurred every 3 months.

Results-Median follow-up for all patients was 7.1 months (range, 1.1-24.3), with 11 patients (73.3\%) followed until death. For the remaining 4 patients alive at the time of analysis, median follow-up was 19.6 months (range, 9.2-24.3). Local control at 6 and 12 months was 91.7 (95\% Confidence Interval [C.I.], 84.6-100.0\%) and 81.5 (95\% C.I., 67.9-100.0\%), respectively. Regional failure was observed in 9 patients $(60.0 \%)$, and 7 patients $(46.7 \%)$ received salvage therapy. Overall survival at 6 months was $60.0 \%$ (95\% C.I., 40.3-88.2\%). Grade 3 or greater

Correspondence: Steven K. M. Lau, M.D., Ph.D., Radiation Oncology PET/CT Center, Moores UCSD Cancer Center, 3855 Health Šxiences Drive, MC 0843, La Jolla, California 92093-0843, steven.lau@utsouthwestern.edu.

Indicates equal contribution

Disclosure: K. T. M. has received honoraria from Varian Medical Systems for giving lectures. The other authors have no personal, financial, or institutional interest in any of the drugs, materials, or devices described in this article. 
treatment-related toxicity was not observed. Median total treatment time was 7.2 minutes (range, 2.8-13.2 minutes).

Conclusion-Single-isocenter, frameless VMAR for multiple intracranial metastases is a promising technique that may provide similar clinical outcomes compared to conventional radiosurgery.

\section{Keywords}

Brain metastasis; frameless; radiosurgery; surface imaging; volumetric modulated arc therapy; single isocenter

\section{Introduction}

Stereotactic radiosurgery (SRS) is a well-established therapy for the management of intracranial metastasis allowing highly conformal delivery of large doses to well-defined target volumes. ${ }^{1}$ Over half of cancer patients who develop brain metastasis present with more than one lesion, ${ }^{2}$ and SRS is routinely used for patients with multiple lesions. Importantly, randomized controlled trials have demonstrated the efficacy of SRS for the treatment of up to four metastases. ${ }^{3-5}$ Moreover, several retrospective studies have described a benefit of SRS for patients with more than four lesions. ${ }^{6-8}$

Several forms of SRS such as Gamma Knife (Elekta, Stockholm, Sweden), CyberKnife (Accuray Inc., Sunnyvale, California), and intensity modulated linear accelerator (linac) based radiosurgery (IMRS) are now widely used. Volumetric modulated arc therapy (VMAT) is a novel treatment paradigm utilizing conventional technology wherein radiation dose is continuously delivered as the treatment gantry rotates. Multileaf collimator (MLC) aperture, gantry rotation speed, and dose delivery rate are simultaneously adjusted with VMAT. ${ }^{9}$ As such, volumetric modulated arc radiosurgery (VMAR) is attractive for its ability to rapidly deliver highly conformal treatments.

SRS for multiple lesions typically employs multiple isocenters, requiring patient repositioning during a single session and sequential treatment of multiple lesions. ${ }^{10,11}$ IMRS allows for treatment planning of multiple lesions using a single-isocenter, thereby eliminating the need for isocenter shifts, significantly reducing treatment times, and enhancing accuracy by reducing the potential for intrafraction patient motion. ${ }^{12}$ Such an approach is advantageous for both patients and clinicians in busy clinics. Importantly, static beam single-isocenter IMRS is associated with clinical outcomes similar to those of traditional SRS methods. ${ }^{13,14}$ Although several dosimetric studies have evaluated the feasibility of VMAR compared to traditional SRS methods, the clinical outcomes associated with VMAR are not well known. ${ }^{15-19}$ Here, we describe our clinical experience using single-isocenter, frameless VMAR for patients with multiple intracranial metastases. To our knowledge, this is the first report of clinical outcomes associated with single-isocenter VMAR for multiple brain metastases. 


\section{Methods}

Retrospective review of radiation oncology records was performed after obtaining institutional review board approval. We previously examined 100 patients with multiple intracranial metastases consecutively treated with single-isocenter, frameless SRS between March 2006 and March 2012 at a single institution. ${ }^{14}$ Patients with intracranial metastatic disease that was histologically confirmed at either the primary or metastatic site were eligible for treatment if they could lie still and tolerate simulation. This report evaluates a subgroup of those patients whose treatment was delivered using VMAT. In total, 15 patients received singleisocenter, frameless VMAR for multiple brain metastases between November 2009 and November 2011 (see Figure, Supplemental Digital Content 1). VMAR was cautiously adopted during this time, as it was considered investigational.

Patient characteristics are summarized in Table 1. Patients had a median of 3 intracranial metastases (range, 2-13). A total of 62 intracranial metastases were treated to a median dose of $20 \mathrm{~Gy}$ (range, 15-30 Gy). The maximum target diameter as determined by contrast enhanced T1-weighted magnetic resonance imaging (MRI) was less than $4.0 \mathrm{~cm}$ in all patients. Three patients (20\%) were treated with more than one fraction. Of these 3 patients, 1 had previously been treated with WBRT, 1 had a brainstem lesion, and 1 had a $3.5 \mathrm{~cm}^{3}$ lesion with a composite PTV of $31.6 \mathrm{~cm}^{3}$. The remaining 12 patients $(80 \%)$ were treated in a single fraction. In total, 3 patients were treated for progressive or recurrent intracranial disease after surgical resection, WBRT, or prior SRS. Two patients (13\%) received WBRT prior to presentation, while the remaining 13 patients $(87 \%)$ did not. Salvage therapy was offered to patients with recurrent local disease or new intracranial metastases and consisted of surgical resection, WBRT, or repeated frameless IMRS.

After obtaining informed consent, patients underwent stereotactic-protocol contrast enhanced T1-weighted brain MRI ( $26 \mathrm{~cm}$ field-of-view, $512 \times 512$ pixel size, $1.5 \mathrm{~mm}$ slice intervals) using a 3.0 Tesla Scanner (General Electric, Fairfield, Connecticut). A detailed description of our patient simulation and setup techniques has been previously published. ${ }^{13,14}$ Notably, we immobilize patients without a bite block in favor of surface image guidance (SIG) in real-time with the AlignRT system (VisionRT Ltd, London, United Kingdom). As previously described, these patients also underwent simulation non-contrast $\mathrm{CT}$ for treatment planning purposes. ${ }^{20}$ During treatment, patient position was continuously monitored with SIG, with a beam hold initiated for deviation exceeding a predefined translational threshold of $1-2 \mathrm{~mm}$ or a rotational threshold of $1^{\circ} .^{20}$

Gross tumor volume and clinical target volume (CTV) for each lesion were identical and defined as the contrast-enhancing volume on axial MRI. Planning target volume (PTV) for each lesion was generated typically by adding a $1 \mathrm{~mm}$ margin to each CTV. Subsequently, a composite PTV was established by adding each individual PTV. For 3 patients, no expansion margin was used. Dose was prescribed to the composite PTV such that each lesion for a given patient was prescribed the same dose. Plan optimization was performed using Eclipse software, version 8.9 (Varian Medical Systems, Palo Alto, California). VMAR planning provided uniform coverage of the PTV and minimal dose to critical structures (Figure 1). Median minimum and maximum PTV coverage was $92 \%$ and $112 \%$, 
respectively. Treatment was delivered using $5 \mathrm{~mm}$ MLC leaves. Radiation Therapy Oncology Group (RTOG) Conformity Index (CI) was defined as the ratio of prescription isodose volume (PIV) to PTV. RTOG Heterogeneity Index (HI) was defined as the ratio of maximum PTV dose to prescription dose. Paddick CI was defined as the ratio of PTV receiving prescription dose squared to the product of PTV and PIV. These representations of conformity have been previously described. ${ }^{21,22}$ Treatment plan characteristics are summarized in Table 2.

All patients completed treatment as planned. Patients were routinely seen one week following VMAR for clinical examination. Thereafter, intracranial progression was assessed by physical evaluation and contrast enhanced MRI every 3 months. Treatment response was analyzed by local control, regional control, and overall survival. Local control was defined as the absence of disease progression, with progression radiographically defined as an increase of greater than $25 \%$ of the bi-dimensional product of the two largest diameters of the lesion. Regional control was defined as the absence of new intracranial metastatic disease occurring outside the treatment volume on radiographic examination. Intracranial status was deemed unknown if radiographic examination had not been obtained by the time of analysis. These patients were excluded from local and regional control analyses but were included in survival analysis. Local control and regional control from the date of treatment were estimated by the Kaplan-Meier method, as was overall survival from the date of diagnosis. Event times were censored at the time of last follow-up for patients without an event at the time of analysis. Toxicity was graded according to the RTOG scale. ${ }^{23}$ Statistical analyses were performed using GraphPad Prism version 6.05 (GraphPad Software, La Jolla, California).

\section{Results}

All patients were treated with single-isocenter, frameless VMAR. A median of $2 \operatorname{arcs}$ (range, 1-4) were used. Arcs were coplanar except in the four patients treated with $\geq 3$ arcs. Three patients were treated using flattening filter-free (FFF) mode. Median treatment time from initial beam on to final beam off was 7.2 minutes (range, 2.8-13.2). Increasing number of lesions (2-4, 5-8, and $\searrow 9$ metastases) was not associated with significantly longer treatment time (data not shown). Median total beam-on time was 5.5 minutes (range, 1.8-8.2).

Dosimetric representations of conformity as described by RTOG CI, RTOG HI, and Paddick CI were generally within accepted limits (Table 2). Median RTOG CI was 1.15 (range, 0.29-2.04). Median RTOG HI was 1.12 (range, 1.05-1.23). Median Paddick CI was 0.77 (range, 0.33-0.90). The only patient with a "major violation" as defined by RTOG CI $<0.9$ or $>2.5$ had 6 very small lesions (cumulative PTV $1.9 \mathrm{~cm}^{3}$ ) treated with a single arc. No other major violations were observed.

Median follow-up for all patients was 7.1 months (range, 1.1-24.3) with 11 patients (73.3\%) followed until death. For the remaining 4 patients alive at the time of analysis, median follow-up was 19.6 months (range, 9.2-24.3).

One patient was excluded from local control analysis owing to unknown intracranial status, and the remaining 14 patients (59 lesions) were evaluable for local control. Of this group, 12 
patients (86\%) and 57 lesions (97\%) were without evidence of local progression at the time of analysis. Local control at 6 and 12 months was $91.7 \%$ (95\% C.I., 84.6-100.0\%) and $81.5 \%$ (95\% C.I., 67.9-100.0\%), respectively (Figure 2). For the 2 patients with local failure, salvage therapy consisted of repeated SRS (1 patient) or no further treatment (1 patient).

Intracranial failure outside the treatment volume was observed in 9 patients $(60.0 \%)$. Regional control at 6 and 12 months was $57.9 \%$ (95\% C.I., 35.8-89.8\%) and 36.2\% (95\% C.I., 8.5-62.4\%), respectively (Figure 3). For the 9 patients with a documented failure, the site of first failure was primarily outside the treatment volume ( 7 patients) rather than only local (1 patient) or simultaneously within and outside the treatment volume (1 patient). Salvage therapy for new intracranial metastases occurring outside of the treatment volume was received by 7 patients (47.0\%) and consisted of repeated SRS (3 patients), WBRT (3 patients), and surgical resection (1 patient).

Overall survival at 6 and 12 months was 60.0\% (95\% C.I., 40.3-88.2\%) and 38.9\% (95\% C.I., 15.6-62.5\%), respectively (Figure 4). The number of patients by RTOG recursive partitioning analysis (RPA) class or who had previously received WBRT were too small to allow for meaningful comparisons stratified by these factors.

Acute treatment-related toxicity occurred in 1 patient (6.7\%). This patient suffered a seizure attributed to cerebral edema responsive to steroids. Late treatment-related toxicity was not observed. Moreover, no grade 3 or greater acute or late treatment-related events were observed. Mean dose to normal brain was $4.2 \mathrm{~Gy}$. Median volume of normal brain receiving $\geq 12 \mathrm{~Gy}$ (V12 Gy) was $38.0 \mathrm{~cm}^{3}$, and median volume of normal brain receiving $\geq 4.5 \mathrm{~Gy}$ (V4.5 Gy) was $350.5 \mathrm{~cm}^{3}$. No discernible relationship between dose to normal brain and toxicity was observed.

\section{Discussion}

SRS is a well-established therapy for the management of intracranial metastasis, with discrete lesions traditionally treated sequentially and treatment duration proportional to the number of isocenters used. We previously reported that static beam single-isocenter IMRS is associated with clinical outcomes comparable to traditional SRS methods. ${ }^{13}, 14,20$ Whereas IMRS is delivered in step-and-shoot fashion using several fixed-position beams, VMAT is delivered continuously as the gantry rotates (see Figure, Supplemental Digital Content 2). VMAT is an attractive technique for rapid, conformal IMRS due to its simultaneous adjustments in MLC aperture, gantry rotation speed, and dose delivery rate. However, the clinical outcomes of this novel technique are not well described. Here, we report, to our knowledge, the first clinical series of single-isocenter, frameless VMAR for multiple intracranial metastases.

\section{Key Results}

In this series, local control at 12 months was $82 \%$, with $97 \%$ of lesions without progression at the time of analysis. Seven patients (47\%) received salvage therapy for new brain metastases outside of the treatment volume. Overall survival at 12 months was $39 \%$. No grade $\geq 3$ toxicity was observed. 


\section{Interpretation}

The clinical outcomes of VMAR for intracranial metastasis are unknown. Local control of $82 \%$ at 12 months in this study is comparable to both frame-based and frameless SRS series. ${ }^{6,14,24,25}$ Unfortunately, the prognosis for patients with intracranial metastasis remains poor, and overall survival at 12 months ranges from $28 \%-45 \%$ with SRS alone in randomized trials. ${ }^{4,5}$ Survival at 12 months of $39 \%$ in the present series compares favorably, particularly when taking into account that $40 \%$ of these patients were RPA class III.

Mayo and colleagues reported a series of 12 patients with a total of 14 intracranial metastases undergoing fractionated VMAR with all but 1 patient receiving 21 Gy in 3 fractions. ${ }^{15}$ The two patients with two brain metastases each in their series were sequentially treated using separate isocenters. At a median follow-up of 3 months, no local failures or adverse events were observed. ${ }^{15}$ In comparison, all 15 patients in the present series had multiple brain metastases treated with a single isocenter, and 12 patients $(80 \%)$ were treated in a single fraction. Thus, the patient population and treatment technique in this report are significantly different compared to the previously described study. Moreover, the radiobiology of single fraction treatment and fractionated treatment may be dissimilar. ${ }^{26}$ All together, single-isocenter, frameless VMAR for multiple brain metastases does not appear to compromise clinical outcomes when compared to traditional SRS techniques.

The feasibility and dosimetric implications of VMAR have been described. First, compared to single-isocenter static beam IMRS, single-isocenter VMAR is associated with reduced monitor units (MUs) and reduced treatment time. ${ }^{18}$ Next, a single-isocenter approach to VMAR rather than using multiple isocenters reduces both MUs and treatment time without compromising conformality. ${ }^{16}$ Finally, the significance of FFF mode in VMAR remains an open question. The dosimetric characteristics of VMAR with or without FFF mode for multiple brain metastases are comparable, although FFF mode provides even greater reductions in treatment time. ${ }^{19,27}$ Taken together, the dosimetric profile of single-isocenter VMAR compares favorably to both conventional frame-based SRS techniques and static beam IMRS. In the present study, dosimetric characteristics of VMAR were generally within accepted standards in concordance with prior feasibility studies. ${ }^{18,19}$

Traditional radiosurgery uses separate isocenters for each lesion which are then treated sequentially, whereas single-isocenter technique allows simultaneous treatment of multiple lesions. Dosimetric characteristics for single-isocenter compared to multiple-isocenter techniques have been described. Single-isocenter strategies have similar conformity with slightly increased dose to non-target tissue compared to multiple-isocenter techniques. ${ }^{16,28}$ Reported clinical outcomes including local control and toxicity are comparable. 13, 14, 20 With traditional multiple-isocenter technique, a given patient is typically repositioned multiple times during the treatment session. Alternatively, a patient may not require repositioning with singleisocenter technique, but accurate setup is critical since both translational and rotational error degrade conformality. ${ }^{29,}{ }^{30}$ At our institution, intrafraction motion after initial setup is minimized by continuous monitoring in real-time with SIG. ${ }^{20}$ 
One consequence of VMAT is the increased volume of non-target tissue receiving low dose as a result of beam angle modulation through gantry rotation. Single-isocenter static beam IMRS is associated with low toxicity. ${ }^{13,14}$ When compared to IMRS, VMAR is associated with a trend for slightly increased low isodose "spill" to surrounding tissue. ${ }^{18}$ Mean dose to normal brain in this series is similar to prior studies of linac-based SRS, ${ }^{13}, 14,18$ but both V12 Gy and V4.5 Gy are higher, as expected, than those published for traditional SRS. ${ }^{31}$ Notably, most patients in this study were treated using one or two arcs, and increasing arc number is associated with superior dosimetric characteristics. ${ }^{31}$ Interestingly, the low isodose spill of four-arc VMAR is similar to traditional frame-based SRS methods using many fixed beam angles. ${ }^{31}$ Increased low isodose spill to non-target brain theoretically could increase the risk of adverse effects. Importantly, minimal toxicity was observed in the present series. Although radionecrosis may be underestimated with standard imaging techniques, ${ }^{32}$ no evidence of symptomatic radionecrosis was detected. While the long-term toxicity of low isodose spill associated with VMAT remains an open question, its clinical significance in patients with multiple intracranial metastases and poor prognosis is unclear.

A significant advantage of our technique is reduced treatment time, and VMAR is one of the most efficient forms of SRS to date. Treatment of multiple intracranial lesions with framebased SRS techniques may last several hours. ${ }^{13}$ Treatment times for multiple-isocenter linac-based SRS often last over 30 minutes, although this can be reduced using a singleisocenter technique. ${ }^{12-14}$ In comparison, patients in this series completed treatment with VMAR for multiple metastases in just over 7 minutes. Reduced treatment time enhances both patient tolerance and provider efficiency, but also reduces intrafraction patient motion. The latter is particularly important for maximizing accuracy and minimizing risk to nontarget tissue, even more so with frameless techniques. ${ }^{33,34}$ Given its efficiency, singleisocenter, frameless VMAR as presented here is a noninvasive treatment strategy for multiple intracranial lesions that appeals to both patients and providers.

\section{Limitations and Generalizability}

As a retrospective analysis study from a single institution, this series is susceptible to all the inherent biases and shortcomings of such analyses. Ultimately, a prospective study would best assess the role of single-isocenter, frameless VMAR for multiple intracranial metastases. At our institution, we gradually implemented single-isocenter VMAR with attention to coverage of PTV and dose to critical structures. Until rigorous studies on this technique are available, it is best performed at a center with expertise using it.

\section{Conclusion}

Results of this study suggest that single-isocenter, frameless SIG VMAR for the simultaneous treatment of multiple intracranial metastases may produce clinical outcomes comparable to both conventional frame-based and other frameless SRS techniques while enhancing patient tolerance and reducing treatment time. Long-term outcomes for VMAR will continue to mature, but this series provides encouraging initial clinical outcomes such that this technique will be used for all intracranial radiosurgery treatments at our institution. 


\section{Supplementary Material}

Refer to Web version on PubMed Central for supplementary material.

\section{Acknowledgments}

This work was supported in part by National Institutes of Health grant PHSGM007198 (UCSD Medical Scientist Training Program). The funders had no role in study design, data collection and analysis, decision to publish, or preparation of the manuscript.

\section{References}

1. Suh JH. Stereotactic radiosurgery for the management of brain metastases. The New England journal of medicine. Mar 25; 2010 362(12):1119-1127. [PubMed: 20335588]

2. Delattre JY, Krol G, Thaler HT, Posner JB. Distribution of brain metastases. Archives of neurology. Jul; 1988 45(7):741-744. [PubMed: 3390029]

3. Andrews DW, Scott CB, Sperduto PW, et al. Whole brain radiation therapy with or without stereotactic radiosurgery boost for patients with one to three brain metastases: phase III results of the RTOG 9508 randomised trial. Lancet. May 22; 2004 363(9422):1665-1672. [PubMed: 15158627]

4. Aoyama H, Shirato H, Tago M, et al. Stereotactic radiosurgery plus whole-brain radiation therapy vs stereotactic radiosurgery alone for treatment of brain metastases: a randomized controlled trial. JAMA : the journal of the American Medical Association. Jun 7; 2006 295(21):2483-2491. [PubMed: 16757720]

5. Kocher M, Soffietti R, Abacioglu U, et al. Adjuvant whole-brain radiotherapy versus observation after radiosurgery or surgical resection of one to three cerebral metastases: results of the EORTC 22952-26001 study. J Clin Oncol. Jan 10; 2011 29(2):134-141. [PubMed: 21041710]

6. Bhatnagar AK, Flickinger JC, Kondziolka D, Lunsford LD. Stereotactic radiosurgery for four or more intracranial metastases. International journal of radiation oncology, biology, physics. Mar 1; 2006 64(3):898-903.

7. Hunter GK, Suh JH, Reuther AM, et al. Treatment of five or more brain metastases with stereotactic radiosurgery. International journal of radiation oncology, biology, physics. Aug 1; 2012 83(5): 1394-1398.

8. Kim CH, Im YS, Nam DH, Park K, Kim JH, Lee JI. Gamma knife radiosurgery for ten or more brain metastases. Journal of Korean Neurosurgical Society. Dec; 2008 44(6):358-363. [PubMed: 19137079]

9. Otto K. Volumetric modulated arc therapy: IMRT in a single gantry arc. Medical physics. Jan; 2008 35(1):310-317. [PubMed: 18293586]

10. Cardinale RM, Benedict SH, Wu Q, Zwicker RD, Gaballa HE, Mohan R. A comparison of three stereotactic radiotherapy techniques; ARCS vs. noncoplanar fixed fields vs. intensity modulation. International journal of radiation oncology, biology, physics. Sep 1; 1998 42(2):431-436.

11. Sankaranarayanan V, Ganesan S, Oommen S, Padmanaban TK, Stumpf J, Ayyangar KM. Study on dosimetric parameters for stereotactic radiosurgery and intensity-modulated radiotherapy. Medical dosimetry : official journal of the American Association of Medical Dosimetrists. Summer;2003 28(2):85-90. [PubMed: 12804705]

12. Lawson JD, Fox T, Waller AF, Davis L, Crocker I. Multileaf collimator-based linear accelerator radiosurgery: five-year efficiency analysis. J Am Coll Radiol. Mar; 2009 6(3):190-193. [PubMed: 19248995]

13. Nath SK, Lawson JD, Simpson DR, et al. Single-isocenter frameless intensity-modulated stereotactic radiosurgery for simultaneous treatment of multiple brain metastases: clinical experience. International journal of radiation oncology, biology, physics. Sep 1; 2010 78(1):91-97.

14. Lau SK, Zhao X, Carmona R, et al. Frameless single-isocenter intensity modulated stereotactic radiosurgery for simultaneous treatment of multiple intracranial metastases. Transl Cancer Res. Aug; 2014 3(4):383-390. [PubMed: 25821723] 
15. Mayo CS, Ding L, Addesa A, Kadish S, Fitzgerald TJ, Moser R. Initial experience with volumetric IMRT (RapidArc) for intracranial stereotactic radiosurgery. International journal of radiation oncology, biology, physics. Dec 1; 2010 78(5):1457-1466.

16. Clark GM, Popple RA, Young PE, Fiveash JB. Feasibility of single-isocenter volumetric modulated arc radiosurgery for treatment of multiple brain metastases. International journal of radiation oncology, biology, physics. Jan 1; 2010 76(1):296-302.

17. Clark GM, Popple RA, Prendergast BM, et al. Plan quality and treatment planning technique for single isocenter cranial radiosurgery with volumetric modulated arc therapy. Practical radiation oncology. Oct-Dec;2012 2(4):306-313. [PubMed: 24674169]

18. Wang JZ, Pawlicki T, Rice R, et al. Intensity-modulated radiosurgery with rapidarc for multiple brain metastases and comparison with static approach. Medical dosimetry : official journal of the American Association of Medical Dosimetrists. Spring;2012 37(1):31-36. [PubMed: 21705211]

19. Wang JZ, Rice R, Mundt AJ, Sandhu A, Murphy KT. Feasibility and advantages of using flattening filter-free mode for radiosurgery of multiple brain lesions. Practical radiation oncology. Oct-Dec;2012 2(4):e165-171. [PubMed: 24674180]

20. Pan H, Cervino LI, Pawlicki T, et al. Frameless, real-time, surface imaging-guided radiosurgery: clinical outcomes for brain metastases. Neurosurgery. Oct; 2012 71(4):844-851. [PubMed: 22989959]

21. Shaw E, Scott C, Souhami L, et al. Single dose radiosurgical treatment of recurrent previously irradiated primary brain tumors and brain metastases: final report of RTOG protocol 90-05. International journal of radiation oncology, biology, physics. May 1; 2000 47(2):291-298.

22. Paddick I. A simple scoring ratio to index the conformity of radiosurgical treatment plans. Technical note. J Neurosurg. Dec; 2000 93(Suppl 3):219-222. [PubMed: 11143252]

23. Cox JD, Stetz J, Pajak TF. Toxicity criteria of the Radiation Therapy Oncology Group (RTOG) and the European Organization for Research and Treatment of Cancer (EORTC). International journal of radiation oncology, biology, physics. Mar 30; 1995 31(5):1341-1346.

24. Sneed PK, Suh JH, Goetsch SJ, et al. A multi-institutional review of radiosurgery alone vs. radiosurgery with whole brain radiotherapy as the initial management of brain metastases. International journal of radiation oncology, biology, physics. Jul 1; 2002 53(3):519-526.

25. Chitapanarux I, Goss B, Vongtama R, et al. Prospective study of stereotactic radiosurgery without whole brain radiotherapy in patients with four or less brain metastases: incidence of intracranial progression and salvage radiotherapy. J Neurooncol. Jan; 2003 61(2):143-149. [PubMed: 12622453]

26. Oermann EK, Kress MA, Todd JV, et al. The impact of radiosurgery fractionation and tumor radiobiology on the local control of brain metastases. J Neurosurg. Nov; 2013 119(5):1131-1138. [PubMed: 24010977]

27. Stieler F, Fleckenstein J, Simeonova A, Wenz F, Lohr F. Intensity modulated radiosurgery of brain metastases with flattening filter-free beams. Radiotherapy and oncology : journal of the European Society for Therapeutic Radiology and Oncology. Dec; 2013 109(3):448-451. [PubMed: 24231243]

28. Vanderspek L, Wang J, Alksne J, Murphy KT. Single fraction, single isocenter intensity modulated radiosurgery (IMRS) for multiple brain metastases: Dosimetric and early clinical experience. International journal of radiation oncology, biology, physics. 2007; 69(3 Suppl):S265.

29. Wang H, Shiu A, Wang C, et al. Dosimetric effect of translational and rotational errors for patients undergoing image-guided stereotactic body radiotherapy for spinal metastases. International journal of radiation oncology, biology, physics. Jul 15; 2008 71(4):1261-1271.

30. Guckenberger M, Roesch J, Baier K, Sweeney RA, Flentje M. Dosimetric consequences of translational and rotational errors in frame-less image-guided radiosurgery. Radiation oncology. 2012; 7:63. [PubMed: 22531060]

31. Thomas EM, Popple RA, Wu X, et al. Comparison of plan quality and delivery time between volumetric arc therapy (RapidArc) and Gamma Knife radiosurgery for multiple cranial metastases. Neurosurgery. Oct; 2014 75(4):409-417. [PubMed: 24871143] 
32. Stockham AL, Tievsky AL, Koyfman SA, et al. Conventional MRI does not reliably distinguish radiation necrosis from tumor recurrence after stereotactic radiosurgery. J Neurooncol. Aug; 2012 109(1):149-158. [PubMed: 22638727]

33. Cervino LI, Pawlicki T, Lawson JD, Jiang SB. Frame-less and mask-less cranial stereotactic radiosurgery: a feasibility study. Phys Med Biol. Apr 7; 2010 55(7):1863-1873. [PubMed: 20224158]

34. Cervino LI, Detorie N, Taylor M, et al. Initial clinical experience with a frameless and maskless stereotactic radiosurgery treatment. Practical radiation oncology. 2012; 2(1):54-62. 2012. [PubMed: 24674037] 

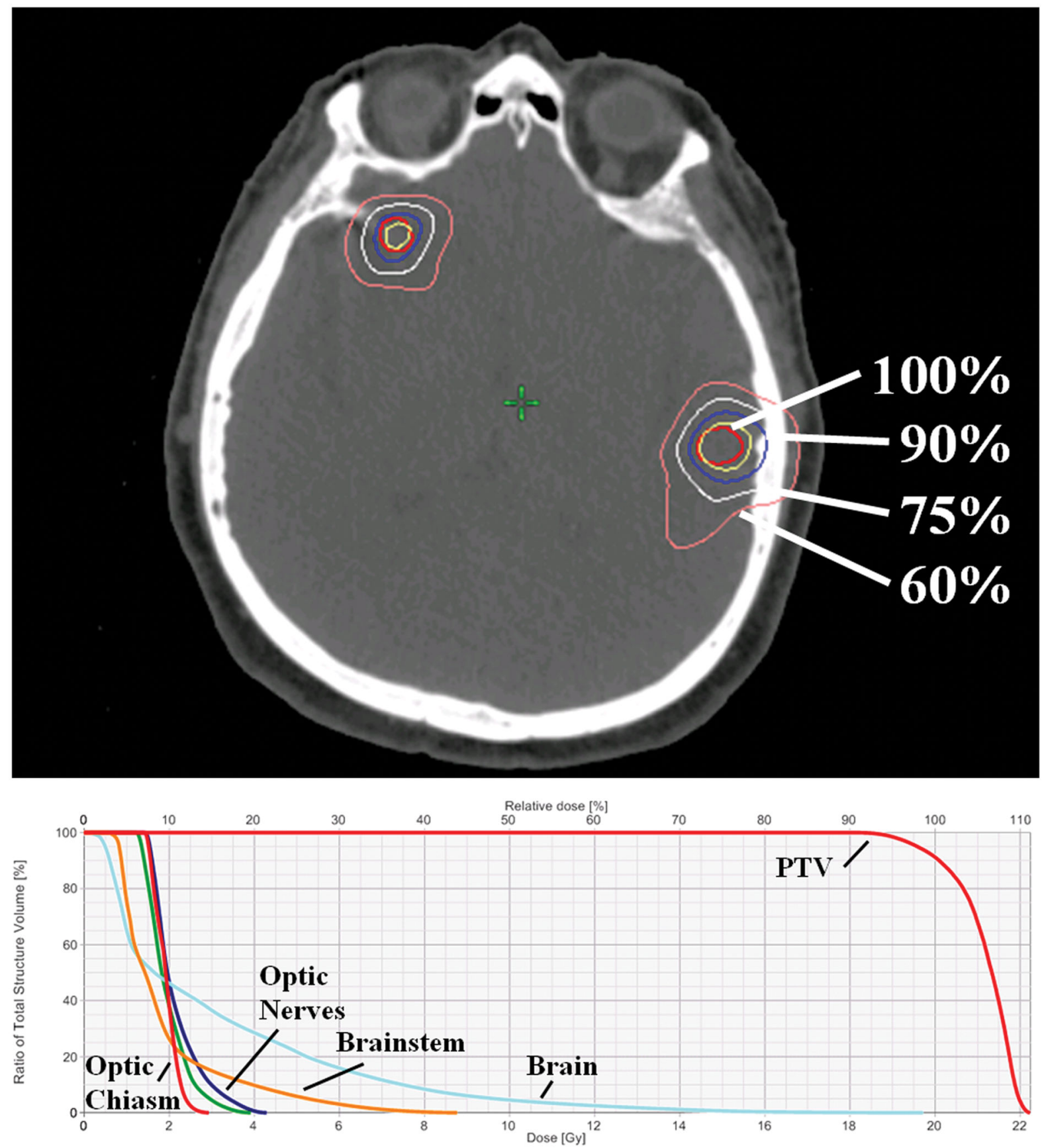

Figure 1.

Representative single-isocenter volumetric modulated arc radiosurgery plan for a patient with three intracranial metastases treated to a prescription dose of $20 \mathrm{~Gy}$. Axial view (top) of the planning CT displaying PTVs and isodose curves. Dose-volume histogram (bottom) displaying doses in absolute (Gy, lower horizontal axis) and relative (\%, upper horizontal axis) measures given composite PTV and normal structures. 


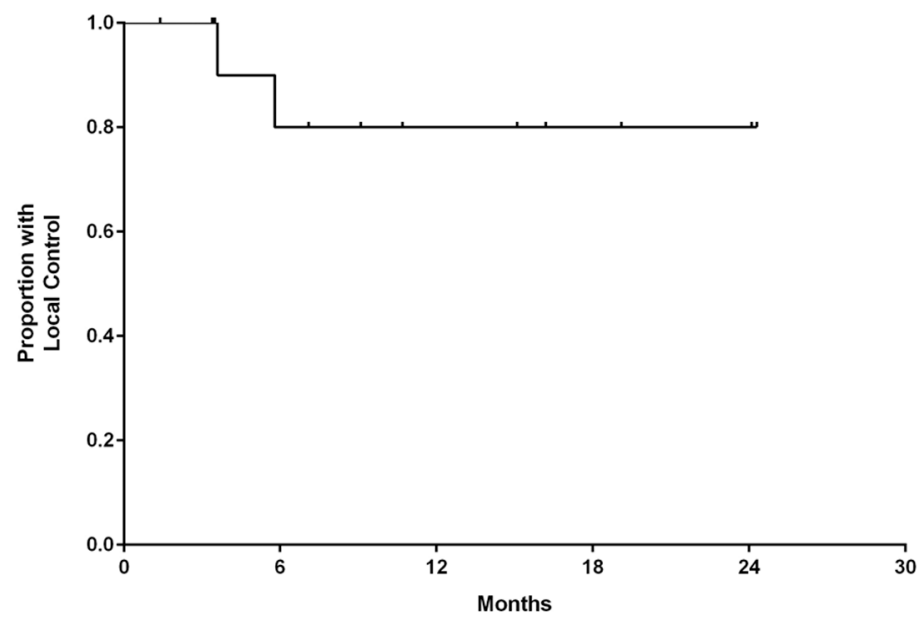

Figure 2.

Kaplan-Meier estimate of local control. 


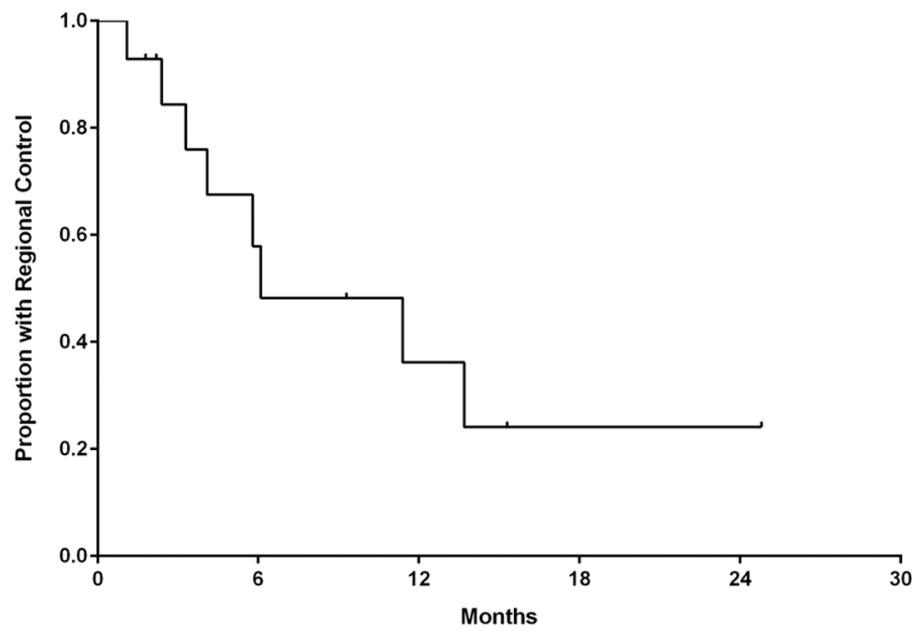

Figure 3.

Kaplan-Meier estimate of regional control. 


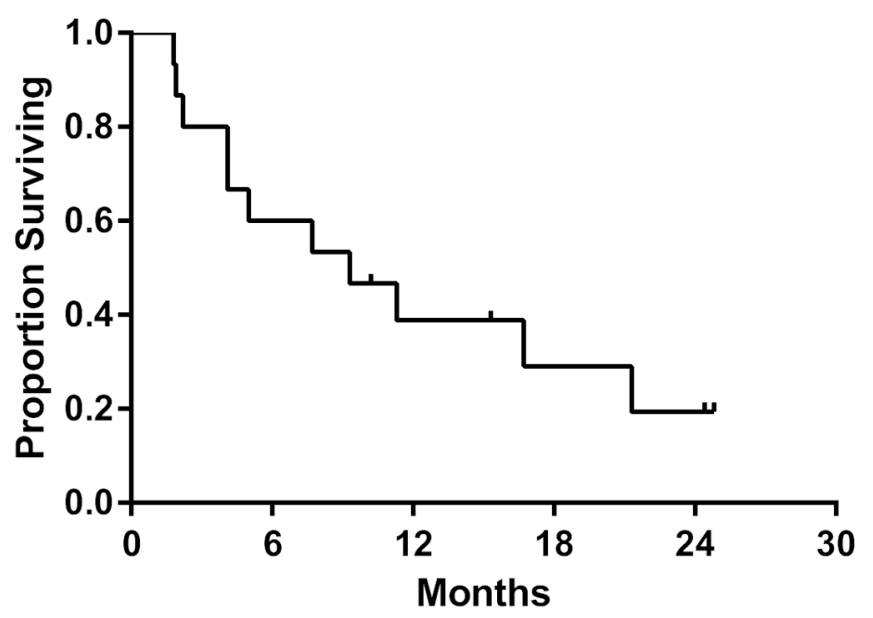

Figure 4.

Kaplan-Meier estimate of overall survival. 


\section{Table 1}

Patient characteristics.

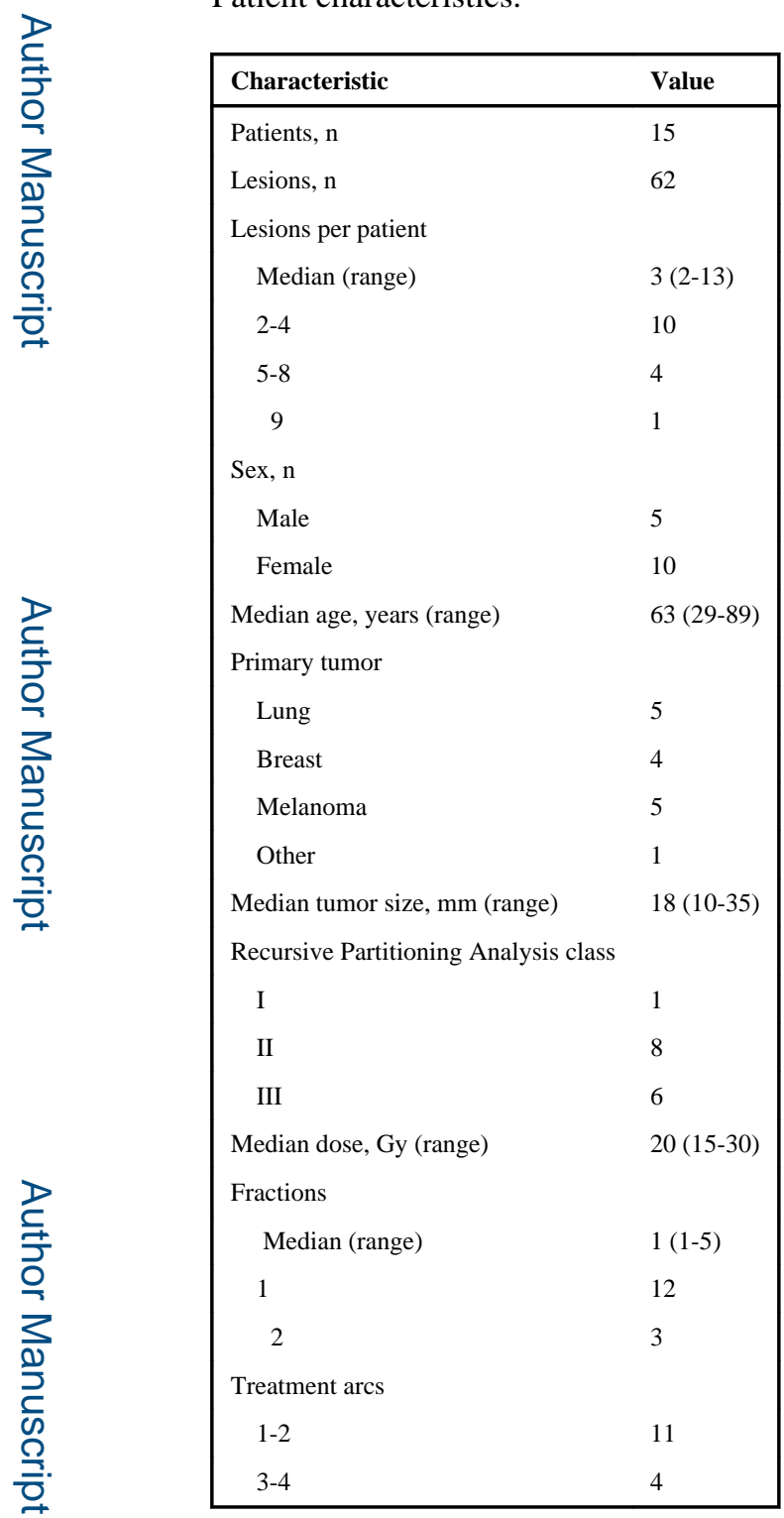


